\title{
Coherent Spectroscopy of PDI-based Artificial Light-Harvesting Antenna
}

\author{
M. Maiuri' ${ }^{1}$, M. B. Oviedo' ${ }^{1}$ M. Koch ${ }^{1}$, M. Myahkostupov' ${ }^{2}$, F. N. Castellano' ${ }^{2}$, G.D. Scholes ${ }^{1}$ \\ ${ }^{I}$ Department of Chemistry, Princeton University, Washingotn Road, Princeton, 08544, New Jersey, United States \\ ${ }^{2}$ Department of Chemistry, North Carolina State University, Raleigh, North Carolina 27695-8204, United States \\ E-mail: gscholes@princeton.edu
}

\begin{abstract}
We time-resolve the energy transfer process in artificial light-harvesting PDI-based tetramers with femtosecond broadband pump-probe anisotropy. We resonantly excite and study the isotropic/anistotropic nuclear motions during the transfer.

OCIS codes: (300.6530) Spectroscopy, ultrafast; (300.6240) Spectroscopy, coherent transient.
\end{abstract}

Artificial light-harvesting systems are suitable models for understanding the role and the structure that govern the early steps following the sunlight capture in Nature. The process of excitation energy transfer (EET) in both natural and artificial systems is ruled by several factors such as the relative distances and orientations between the chromophores, their energetic levels and electronic couplings [1-2]. Many works have explored these features by studying several type of chromophores (i.e. chlorophylls, porphyrins, phthalocyanines, etc.) linked together via molecular scaffolds or bonds [2-5]. Among those, perylenebis(dicarboximide)s (PDI)s represent suitable model systems for fundamental studies of photoinduced energy transfer processes and charge separation mechansisms for light harvesting and photovoltaics application respectivley owing to their high photostability and tendancy to self-assemble in solution, mostly by $\pi-\pi$ stacking. $[2,6]$.

Here we studied PDI-based tetramer supramolecule recently synthetized by Castellano and coworkers (Figure 1a) [7]. The chromophoric monomer subunit is constituted by a bay-substituted $\pi$-conjugated phenylethynyl-perylenediimides (PDICCPh) and the antenna-tetramers are synthetized via methane-center. The conjugated scaffolds fix the spatial orientations of the chromophores with respect to one another. Broadband pump-probe spectroscopy and polarization anisotropy decay analysis were impelmented in conjuction with density functional theory (DFT) calculations to excite and assign Franck-Condon active vibrations present in the antenna supramolecules. This methodology was initially applied to the PDICCPh monomer to identify the active vibrations giving rise to vibrational quantum coherence. This analysis is crucial for revealing possible vibronic interactions during the EET dynamics in the tetramers. The assignment of the vibrational coherences present in the PDI subunits to specific nuclear coordinates will offer us tools to evaluate possible bridge-design principles that can influence the energy transfer (either coherently or incoherently) [8,9].

Figure $1 \mathrm{~b}$ shows the measured ground state absorption spectrum for the PDICCPh monomer. It is characterized by a strong absorption peak at $545 \mathrm{~nm}$ and is dominated by Franck-Condon progressions that partition the transition dipole into a hierarchy of vibronic resonances. The molecular structure of PDICCPh (inset in Figure 1b) was optimized at the DFT level without symmetry constraints using the Gaussian 09 code [10]. The B3LYP exchange correlation functional was employed, and the 6-311G(d) basis set was used for the expansion of the Kohn-Sham orbitals of all the atoms [10]. From the computed results, it is clear that the PDI core unit preserves the $\pi$-delocalization throughout the molecule, and the inclusion of long hydrocarbon chains and phenylethyne does not affect the $\pi$-conjugation. At the optimized geometry, time-dependent DFT (TDDFT) calculations were performed to compute the excited-state vertical transition energies and oscillator strengths. In Figure 1c are reported the electronic excitations and the corresponding energy values, calculated at the TD-DFT/B3LYP/6-311G(d) level of theory, in good agreement with the measured absorption spectrum. The corresponding frontier molecular orbitals (HOMO1, HOMO, LUMO and LUMO+2) involved are depicted in Fig. 1d. From these results it can been seen that the main electronic excitations are $\pi-\pi *$ transitions. These preliminary and promising calculations on the PDI monomer will be extended to the characterization of the geometrical arrangement of the tetramer and will serve as reference for the assignement of vibrational coherences observed in the pump-probe experiments and here reported.

We analyzed and compare the vibrational coherences in both the PDICCPh monomer and Tetramer exploiting our broadband pump-probe apparatus pumped by a home-built NOPA (16-fs pulse duration) spanning the visible frequency range [11]. To evaluate the EET process, pump-probe polarization anisotropy measurements have been performed, collecting the polarization anisotropy map $\mathrm{r}(\lambda, \mathrm{t})$. It is defined as: $\mathrm{r}(\lambda, \mathrm{t})=\left(\Delta \mathrm{T} / \mathrm{T}(\lambda, \mathrm{t})_{\|}-\Delta \mathrm{T} / \mathrm{T}(\lambda, \mathrm{t})_{\perp}\right) /\left(\Delta \mathrm{T} / \mathrm{T}(\lambda, \mathrm{t})_{\|}+2 \Delta \mathrm{T} / \mathrm{T}(\lambda, \mathrm{t})_{\perp}\right)$, where $\Delta \mathrm{T} / \mathrm{T}(\lambda, \mathrm{t}) \|$ and $\Delta \mathrm{T} / \mathrm{T}(\lambda, \mathrm{t})_{\perp}$ are the differential transmission signals measured in parallel and perpendicular pump/probe polarization configurations. This technique monitors the temporal decay of the transition dipole moment direction following photo-excitation: upon EET to adjacent PDIs, the change in excited transition dipole moment direction can be related to the EET rate between the PDIs in the tetramer. Owing to the short pulse duration 
we were able to impulsively excite the main vibration coupled with the electronic transitions as they are revealed as coherent oscillations superimposed on the pump-probe traces.

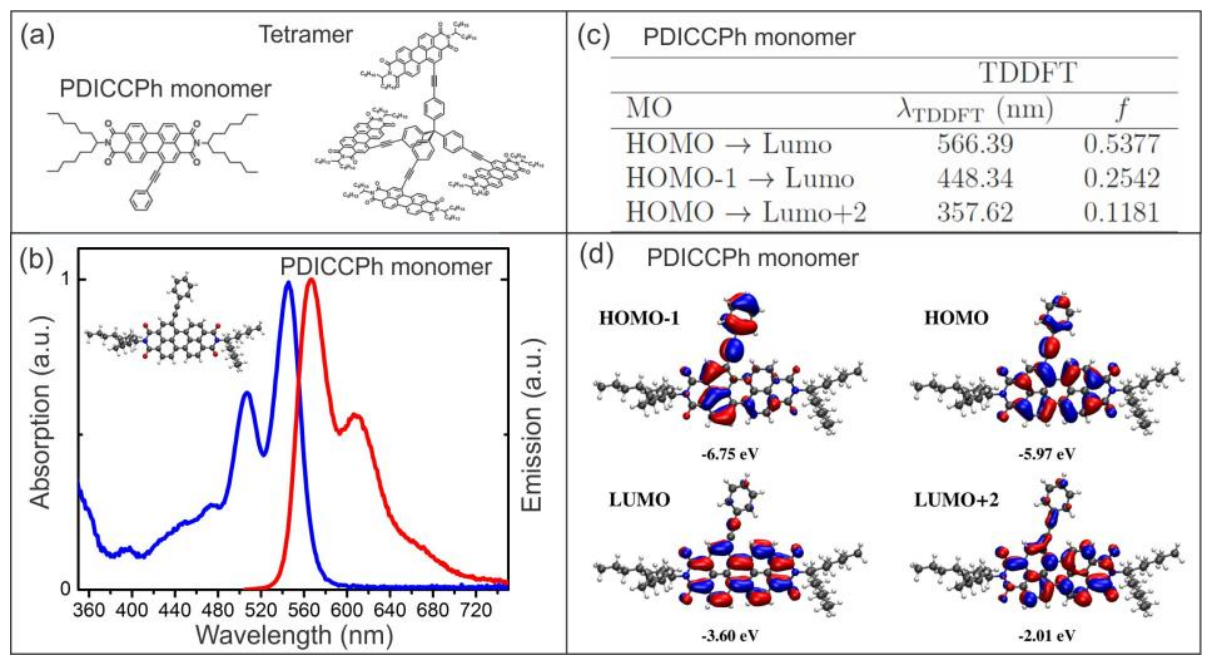

Fig. 1. (a) Chemical structure of the reference monomer PDICCPh and the tetramer (b) Steady state absorption (blue) and emission (red) of the PDI monomer in DCM solvent, inset: DFT optimized geometry of the PDI using the B3LYP functional and 6-311G(d) basis set; (c) Absorption energies of PDI in vacuum calculated by linear response (LR) TD-DFT/B3LYP/6-311G(d); (d) Graphical representation of the highest occupied and lowest unoccupied molecular orbitals of PDI obtained by performing a TD-DFT/B3LYP/6-311G(d) calculation.

Figure 2 compares the Tetramer and the PDICCPh monomer results. Figure 2a (right panels) reports the $\Delta \mathrm{T} / \mathrm{T}$ pump-probe signals measured at magic angle and the corresponding residual oscillations obtained after a bi-exponential fit. A selected probe wavelength at $600 \mathrm{~nm}$ (in the fluorescence spectral region) was chosen to show the residual oscillations. The traces (black dots in Fig. 2a) were fitted with a sum of four damped cosines (red lines in Fig. 2a): two oscillatory functions are needed to fit the strong off resonant solvent modes at $280 \mathrm{~cm}^{-1}$ and $700 \mathrm{~cm}^{-1}$; the fits revealed two main oscillations from the PDI with resonances at $530 \mathrm{~cm}^{-1}$ and $1360 \mathrm{~cm}^{-1}$ wavenumber with dephasing times of $0.09 \mathrm{ps}$ and $1.3 \mathrm{ps}$ respectively. Similar fitting values were found for the monomer and the tetramer. The corresponding FourierTransformed (FT) spectrum is depicted in Fig 2c (red curves). The FT spectra of the tetramer follows closely the one of the monomer, suggesting that the main PDI normal modes (ca. $1360 \mathrm{~cm}^{-1}$ and $500 \mathrm{~cm}^{-1}$, assigned to the main perlyene core skeleton motion [12]) are preserved in the tetramer. This is probably due the arrangements of the subunits in the tetramers where the PDIs are displaced far enough that the nuclear motions are not altered by the interactions with the other chromophores (weak coupling).

Interestingly, the results of the anisotropy pump-probe map $r(\lambda, t)$, calculated as describe above and reported in Fig $2 b$, shows that coherent oscillations are preserved in the polarization anisotropy decay. Figure 2c shows the anisotropy map and a selected probe trace in the two samples. The corresonding FT spectra (blue traces in Fig 2c) are plotted against the FT spectra recorded in the isotropic condition (red traces in Fig. 2c) in Fig. 2c. It is evident that the resonances are different compared to the magic angle case, proving that pump-probe anisotropy can be a useful tool to reveal vibrational resonances associated with anisotropic nuclear modes and asymmetry, such as a clear appearance of the C-C triple bond stretch around $2100 \mathrm{~cm}^{-1}$ from the asymmetric phenylethyne group. Our preliminary DFT calculations on the PDICCPh monomer shows that the frequencies around $1300 \mathrm{~cm}^{-1}$ correspond to in-plane vibrational mode (Fig. 2d), which correlates with the experimental data.

The decay of the anisotropy value in the tetramer is taken as the evident sign of the EET in contrast with the monomer constant anisotropy value. For sake of completeness we measured the full anisotropy decay up to ns-timescale (here not shown) with a commercial pump-probe system and narrowband pump excitation. We extracted the $\mathrm{K}_{\mathrm{EET}}$ rate constant of $1 / 4.7 \mathrm{ps}$ for the Tetramer, in agreement with recent findings on similar tetramers [13]. Such anisotropy measurements also reveal decay features due to rotational diffusion on the nanosecond timescale (ca. $260 \mathrm{ps}$ for the monomer and $400 \mathrm{ps}$ for the te tramer).

The interaction of the active vibrational modes and the electronic coupling in the tetramer will be deeply studied with further calculations and 2D electronic spectroscopy. 
(a) $520 \quad 560 \quad$ Probe Wavelength $(\mathrm{nm})$
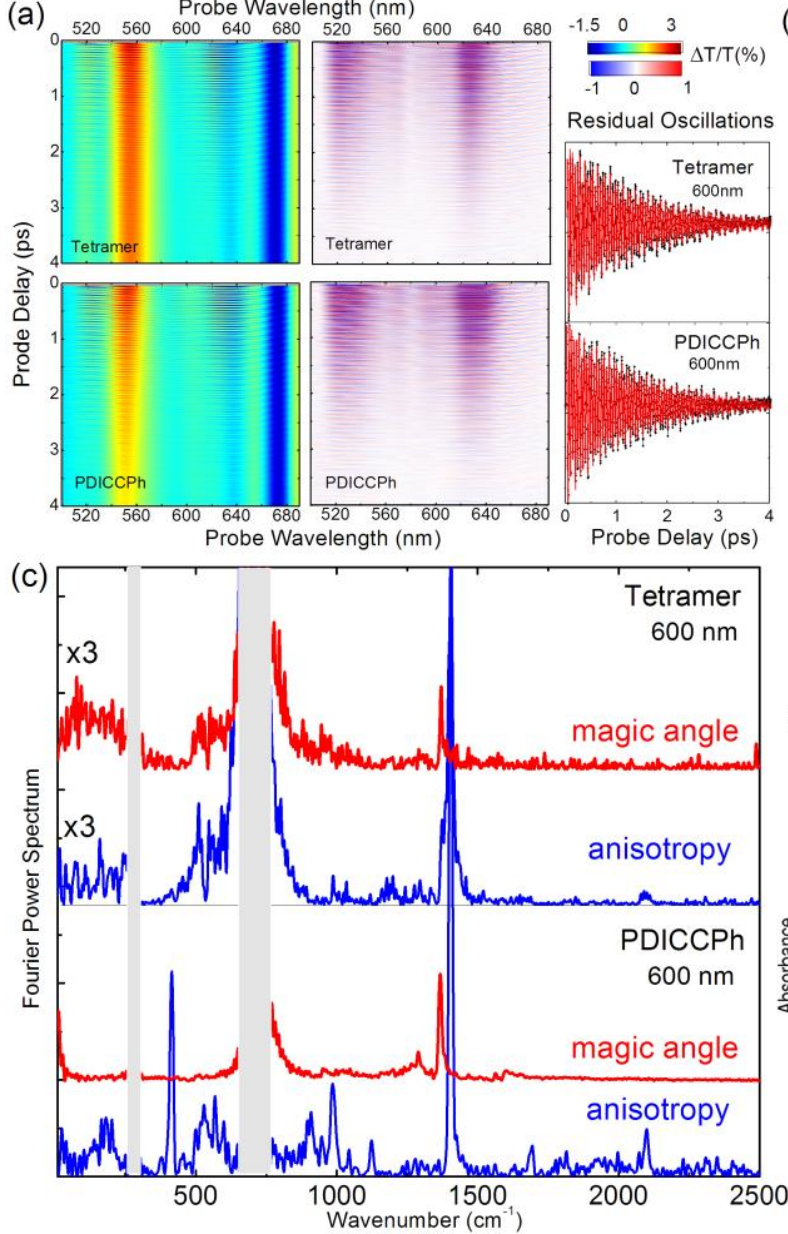

(b)
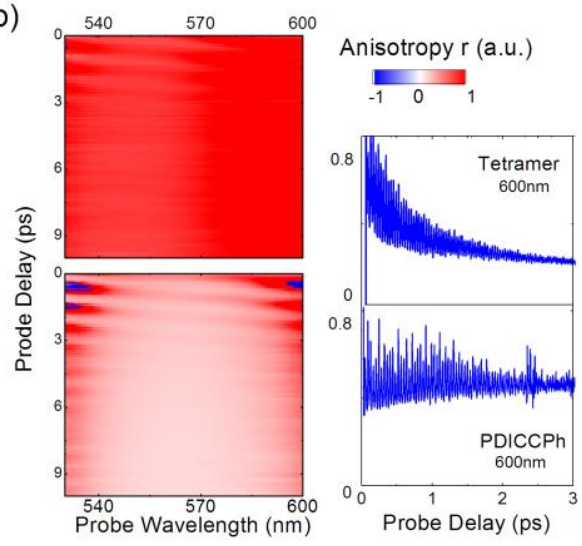

(d) PDICCPh ground state normal modes
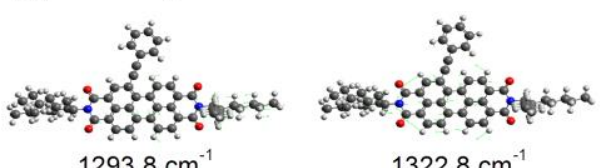

$1293.8 \mathrm{~cm}^{-1}$

$1322.8 \mathrm{~cm}^{-1}$

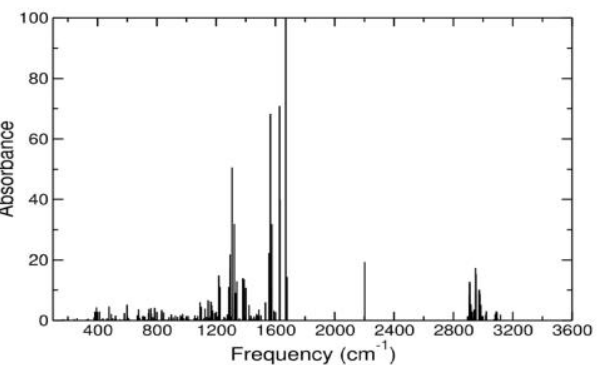

Fig. 3. (a) Left panels: $\triangle T$ T T pump-probe maps of Tetramer (upper) and PDICCPh monomer (lower) in DCM and corresponding oscillatory maps after subtraction of a bi-exponential fit at magic angle configurations. Selected residual oscillations at $600 \mathrm{~nm}$ probe wavelength (back dots) fitted with four damped cosines (red solid traces). (b) Polarization anisotropy maps calculated from $\Delta \mathrm{T} / \mathrm{T}$ parallel/perpendicular experiments and corresponding decay at $600 \mathrm{~nm}$. (c) Corresponding FT Power spectrum for the magic angle experiment and the anisotropy oscillations. Grey squares indicates the DCM solvent strong modes. (d) Two of the normal ground state modes calculated from DFT for the PDICCPh monomer.

[1] R. E Blankenship, Molecular Mechanisms of Photosynthesis; Blackwell Science: Oxford, U.K., (2002).

[2] M. R Wasielewski, "Self-Assembly Strategies for Integrating Light Harvesting and Charge Separation in Artificial Photosynthetic Systems," Acc. Chem. Res. 42, 1910-1921 (2009).

[3] V. L. Gunderson, S. M. M. Conron, M. R. Wasielewski, "Self-Assembly of a Hexagonal Supramolecular Light-Harvesting Array from Chlorophyll a Trefoil Building Blocks. Chem. Commun. (Cambridge,U.K.) 46, 401-403 (2010).

[4] E. Hindin, R. A. Forties, R. S. Loewe, A. Ambroise, C. Kirmaier, D. F. Bocian, J. S. Lindsey, D. Holten, R. S. Knox, "Excited-State Energy Flow in Covalently Linked Multiporphyrin Arrays: The Essential Contribution of Energy Transfer between Nonadjacent Chromophores," J. Phys. Chem. B, 108, 12821-12832 (2004).

[5] X. Li, L. E. Sinks, B. Rybtchinski, M. R. Wasielewski, "Ultrafast Aggregate-to-Aggregate Energy Transfer within Self-Assembled LightHarvesting Columns of Zinc Phthalocyanine Tetrakis- (Perylenediimide)". J. Am. Chem. Soc., 126, 10810-10811 (2004).

[6] M. R. Wasielewski, "Photoinduced Electron Transfer in Supramolecular Systems for Artificial Photosynthesis," Chem. Rev. 92 435-461 (1992).

[7] M. Myahkostupov, F. N. Castellano, "Tetrahedral rigid core antenna chromophores bearing bay substituted perylenediimidies" Tetrahedron, 71, 9519-9527 (2015).

[8] T. Forster, In Modern Quantum Chemistry; O. Sinanoglu, ed. (Academic, New York, NY, 1965).

[9] A. Kolli, E. J. O'Reilly, G. D. Scholes, A. Olaya-Castro," The fundamental role of quantized vibrations in coherent light harvesting by cryptophyte algae," J. Chem. Phys. 137, 174109-15 (2012).

[10] M. J. Frisch, G.W. Trucks, H.B. Schlegel, G.E. Scuseria, M.A. Robb, J.R. Cheeseman, et al. Gaussian 09, revision C.01. Wallingford, CT: Gaussian, Inc.; (2009).

[11] S. D. McClure, D. B. Turner, P. C. Arpin, T. Mirkovic, G. D. Scholes," Coherent oscillations in the PC577 cryptophyte antenna occur in the excited electronic state," J. Phys. Chem. B,118, 1296-1308, (2014).

[12] K. E. Brown, B. S. Veldkamp, D. T. Co, M. R. Wasielewski, "Vibrational Dynamics of a Perylene-Perylenediimide Donor-Acceptor Dyad Probed with Femtosecond Stimulated Raman Spectroscopy,” J. Phys. Chem. Lett., 3, 2362-2366 (2012).

[13] C. Ramanan, C. H. Kim, T. J. Marks, M. R. Wasielewski, "Excitation Energy Transfer within Covalent Tetrahedral Perylenediimide Tetramers and Their Intermolecular Aggregates,”J. Phys. Chem. C, 118, 16941-16950 (2014). 